\title{
AÇÃO CICATRIZANTE DE PLANTAS MEDICINAIS: UM ESTUDO DE REVISÃO
}

\author{
Danieli A B Moreski ${ }^{1 *}$ \\ Eneri Vieira de Souza Leite-Mello ${ }^{1}$ \\ Fernanda Giacomini Bueno ${ }^{2}$
}

MORESKI, D. A. B.; LEITE-MELLO, E. V. de S.; BUENO, F. G. Ação cicatrizante de plantas medicinais: um estudo de revisão. Arq. Cienc. Saúde UNIPAR, Umuarama, v. 22, n. 1, p. 63-69, jan./abr. 2018.

\begin{abstract}
RESUMO: Plantas Medicinais são utilizadas desde a antiguidade para tratar uma série de doenças desempenhando um papel fundamental na saúde mundial. O uso da fitoterapia é muitas vezes o único recurso terapêutico de algumas comunidades. A Organização Mundial da Saúde reconhece a fitoterapia como uma alternativa de tratamento viável com baixo custo e recomenda o levantamento, identificação, estímulo e orientação do uso das plantas medicinais que possuem eficácia e segurança terapêutica comprovada. Nos últimos anos, os fitoterápicos ganharam espaço importante na reparação de tecidos, obtendo a partir deles formulações que agem no tratamento das feridas. O presente estudo teve por objetivo analisar pesquisas envolvendo plantas medicinais e seu poder cicatrizante. Foram utilizados dados de bases online, dos últimos dez anos, utilizando como descritores: "feridas, cicatrização, plantas medicinais". Foram analisados os nomes científico e popular das plantas, a parte da planta que foi utilizada para os experimentos, tipo de extração e utilização, classes das substâncias e efeito na cicatrização. As plantas pesquisadas foram: Aloe vera (L.) Burm. f. (Babosa), Coronopu didymus (Mastruz), Tabebuia avellanedae (Ipê roxo), Arnica Montana L. (Arnica), Orbignya phalerata (Babaçu), Stryphnodendron adstringens Martius (Barbatimão), Caesalpinia ferrea Martius (Jucá), Chenopodium ambrosioides L. (Erva de Santa Maria), Triticum vulgare (Trigo), Tabernaemontana catharinensis (Jasmim), Calendula officinalis (Calêndula) e Helianthus annus (Girassol), Catharanthus roseus L. (Vinca Rosea) e Schinus terebinthifolius Raddi (Aroeira). Assim, pode-se observar que todas as plantas estudadas no artigo favoreceram o processo de cicatrização por meio de diferentes atividades, e que essas atividades estão relacionadas com as diversas classes de metabólitos.
\end{abstract}

PALAVRAS CHAVES: Cicatrização. Feridas. Plantas medicinais.

\section{HEALING ACTION OF MEDICINAL PLANTS: A REVIEW}

\begin{abstract}
Medicinal plants have been used to treat a number of diseases since ancient times, and play a key role in human health. In recent decades, modern medicine has made great advances, but plants still contribute to the development of medicines. The use of herbal medicine is often the only therapeutic resource available in some communities. The World Health Organization recognizes herbal therapy as a viable treatment alternative at a low cost and recommends the collection, identification, stimulation and guidance of the use of medicinal plants that have presented efficacy and safety therapeutics. In recent years, herb therapy has gained important space in the repair of tissues through formulations that act in the treatment of wounds. The aim of this study was to analyze studies involving medicinal plants and their healing potential. Data from online databases from the past ten years were analyzed, using the descriptors: "wounds, healing, medicinal plants". The scientific and popular names of plants, the part of the plant used, type of extraction, therapeutic use, classes of substances and effect on wound healing were analyzed. The plants studied were: Aloe vera (L.) Burm. f. (Babosa), Coronopu didymus (lesser swine cress), Tabebuia avellanedae (pink ipe), Arnica Montana L. (wolf's bane), Orbignya phalerata (Babassu), Stryphnodendron adstringens Martius (Barbatimão), Caesalpinia ferrea Martius (Brazilian ironwood), Chenopodium ambrosioides L. (wormseed), Triticum vulgare (wheat), Tabernaemontana catharinensis (Jasmin), Calendula officinalis (Calendula) and Helianthus annus (sunflower), Catharanthus roseus L. (Madagascar periwinkle) and, Schinus terebinthifolius Raddi (Brazilian peppertree). Thus, the results showed that all the studied plants favored the wound healing process through different mechanism. These activities may be related to the different classes of substances. KEYWORDS: Medicinal Plants. Wound healing. Wounds.
\end{abstract}

\section{Introdução}

A pele é o maior órgão do corpo humano e ocupa lugar de destaque na esfera psíquica dos indivíduos, representando o elo indivíduo-sociedade-ambiente físico (ROTTA, 2008), representando $15 \%$ de seu peso corporal. Reveste e delimita o organismo desempenhando funções de defesa contra agressões químicas, físicas e biológicas. A pele tem como função vital a conservação da homeostasia, desempenhando funções sensoriais. É composta de duas camadas: a epiderme, mais externa, constituída por um epitélio estratificado pavimentoso queratinizado e, uma camada interna de tecido conjuntivo, a derme, onde são encontrados vasos e nervos. Abaixo da derme encontra-se a hipoderme, servindo de união aos órgãos subjacentes. Os pêlos, unhas e glândulas sudoríparas e sebáceas são estruturas anexas da pele (OVAL-

\section{LE; NAHIRNEY, 2008).}

Todos os animais apresentam mecanismos eficientes de reparação tecidual que promovem à re-epitelização da epiderme e a substituição da derme por nova matriz extracelular. A reparação tecidual é um processo dinâmico que envolve mediadores solúveis, elementos sanguíneos e matriz extracelular. A capacidade de reparo dos tecidos danificados nos seres vivos é fascinante, pois ao sofrer um trauma, a pele inicia um processo complexo, gradativo e sistêmico que pode durar até dois anos envolvendo processos de hemostasia, inflamação e reparação (CARNEIRO; RAMOS-E-SILVA, 2007).

Fundamentalmente, o processo de cicatrização se dá em três fases: inflamação, proliferação e remodelação (BALBINO et al., 2005). A cicatrização de feridas é resumida em uma organização de eventos celulares e moleculares

DOI: 10.25110 /arqsaude.v22i1.2018.6300

${ }^{1 *}$ Departamento de Ciências Morfológicas, Programa de Pós-Graduação (Lato Sensu) em Anatomia e Histologia: Métodos de ensino e Pesquisa, Universidade Estadual de Maringá, Maringá, Brasil.

${ }^{2}$ Centro de Ciências Médicas e Farmacêuticas, Universidade Estadual do Oeste do Paraná, Cascavel, Brasil.

*Endereço para correspondência: Rua: Faustino dos Santos Souza, nº 21, CEP: 8717-5000 - Itambé/PR. E-mail: danielibobi@hotmail.com 
que agem reciprocamente para reconstituir o tecido. A fase inflamatória ocorre assim que o tecido é lesionado resultando em inchaço, rubor, calor e dor. Após a ocorrência de um trauma, ocorre o extravasamento sanguíneo que preenche o local lesado com plasma e elementos celulares. Intimamente ligada à fase de coagulação, a inflamação depende, além de inúmeros mediadores químicos, das células inflamatórias, como os leucócitos polimorfonucleares (PMN), macrófagos e linfócitos (MANDELBAUM et al., 2003). Os polimorfonucleares chegam ao momento da irritação do tecido permanecendo por um período que varia de três a cinco dias. Essas células são responsáveis pela fagocitose das bactérias. Neta fase, as células mais importantes, os macrófagos, permanecem do terceiro ao décimo dia no local da ferida (DIEGELMANNET al., 1981). A fase inflamatória também conta com o papel da fibronectina que se prende simultaneamente à fibrina e ao colágeno, funcionando como uma "cola", consolidando assim o coágulo de fibrina (GRINNELL et al., 1981).

A segunda fase, proliferativa, é responsável pela re-epitelização (recobrimento da ferida por um novo epitélio) e a formação do tecido de granulação (tecido conjuntivo vascularizado) (SINGER; CLARK, 1999). A re-epitelização ocorre a partir das células da camada basal onde os queratinócitos, com a lesão, iniciam o processo mitótico e proliferam em direção ao centro da ferida. A formação do tecido de granulação (por volta do quarto dia) ocorre a partir da liberação de fatores de crescimento e quimiotáticos pelas plaquetas e macrófagos, os quais estimulam a migração e ativação intensificada dos fibroblastos. Estas células são essenciais para a formação do tecido de granulação por serem responsáveis pela produção de colágeno local juntamente com a neovascularização do tecido (angiogênese), fenômeno conhecido como fibroplasia (MANDELBAUM et al., 2003; BALBINO et al., 2005). O tecido de granulação é formado por células tais como fibroblastos, macrófagos, componentes neovasculares e da matriz (glicosaminoglicanas, fibronectina e colágeno) (MANDELBAUM et al., 2003). É um tecido edematoso formado por vasos imaturos que se rompem facilmente.

Por volta do décimo dia, a ferida encontra-se toda preenchida pelo tecido de granulação, neovascularizada e com presença de fibras colágenas, iniciando-se a última fase, de remodelamento (MANDELBAUM et al., 2003; BALBINO et al., 2005). Nesta fase, o tecido continua a ser enrijecido por mais fibras colágenas obtendo aparência de cicatriz (BALBINO et al., 2005). Esta fase de reparação tecidual compreende a remodelação da matriz extracelular e a maturação da neoepiderme nas margens das grandes feridas. Enquanto o tecido de granulação invade o espaço mais central da ferida, a matriz extracelular das margens se diferencia, qualitativa e quantitativamente, daquela situada no centro (CARNEIRO; RAMOS-E-SILVA, 2007). O colágeno é responsável pela resistência e integridade do tecido e dos novos vasos dependendo do tipo e quantidade das fibras formadas. Estas fibras são classificadas como tipo I e III de acordo com o grau de maturação, sendo a sua quantificação essencial para se comparar a reparação tecidual. A fibra colágena tipo I é considerada a fibra madura e a tipo III a fibra jovem (CUTTLE et al., 2005). A quantidade de colágeno produzida e a forma como as fibras estão organizadas determina a resistência do novo tecido formado (BALBINO et al., 2005).
Ao final desta fase, os anexos da pele (folículos pilosos e glândulas) começam a aparecer na parte cicatrizada.

Em alguns casos de feridas cutâneas há a dificuldade em sua cicatrização, devido a estados patológicos associados como a diabetes, perturbações-imune, isquemia e estase venosa e, em ferimentos, tais como queimadura. A diabetes prejudica numerosos componentes de cicatrização de feridas, incluindo hemostasia e inflamação, matriz deposição e angiogênese. Estas deficiências estão presentes em uma ampla variedade de tecidos, incluindo a pele. Assim, a busca de medicamentos que possam acelerar esse processo cicatricial vem aumentando. Uma alternativa é o uso de plantas medicinais, por serem muito utilizadas desde o inicio da humanidade no tratamento de feridas. A importância das plantas para a saúde dos seres vivos reside no fato de que elas são à base da maioria dos tratamentos ministrados ao redor do mundo (CANDIDO, 2001). Atualmente já se conhece os efeitos de várias plantas medicinais que ajudam na cicatrização da pele lesada. Assim, este trabalho teve como objetivo reunir dados sobre plantas medicinais que atuam no processo de cicatrização de feridas cutâneas por meio de revisão bibliográfica.

\section{Metodologia}

O levantamento bibliográfico foi realizado nas bases de dados online SCIELO, GOOGLE SCHOLAR, SCIENCEDIRECT, LILACS, PUBMED, utilizando como descritores: "feridas, cicatrização, plantas medicinais". No entanto, foram utilizados dados de resultados publicados nos últimos dez anos. Após escolha dos artigos partiu-se para a construção dos resultados.

As plantas pesquisadas foram: Aloe vera (L.) Burm. f. (Babosa) (FALEIRO et al., 2009), Coronopu didymus (Mastruz) (NITZ et al., 2006), Tabebuia avellanedae (Ipê roxo) (COELHO et al., 2010), Arnica montana L. (Arnica) (NASCIMENTO, 2011), Orbignya phalerata (Babaçu) (AMORIM et al., 2006), Stryphnodendron adstringens Martius (Barbatimão) (COELHO et al., 2010), Caesalpinia ferrea Martius (Jucá) (OLIVEIRA et al., 2010), Chenopodium ambrosioides L. (Erva de Santa Maria) (RIBEIRO, 2011), Triticum vulgare (Trigo) (Souza et al., 2006), Tabernaemontana catharinensis (Jasmim) (JANNING et al., 2011), Calendula officinalis (Calêndula) e Helianthus annus (Girassol) (WENDT, 2005), Catharanthus roseus L. (Vinca Rosea) (Nayak \& Pereira, 2006) e Schinus terebinthifolius Raddi (Aroeira) (NUNES et al., 2006).

\section{Resultados}

O uso de plantas medicinais é tão antigo quanto à espécie humana e é muitas vezes o único recurso de muitas comunidades e grupos étnicos, sendo a mesma comercializada em feiras livres de rua, mercados populares e fundo de quintal (AMOROZO; GELY, 1988). Estas plantas medicinais, nos últimos anos, ganharam espaço importante na reparação do tecido, incorporados à formulações que agem melhorando o tratamento das feridas.

Dentre estas plantas foi encontrado o Orbignya phalerata, popularmente conhecido como "babaçu”. É uma palmeira, pertencente à família Palmae, nativa do meio-norte do Brasil, com prevalência no Estado do Maranhão (SILVA, 
1995). O mesocarpo do babaçu é conhecido por sua ação anti-inflamatória (SILVA, 2001). Além disso, tem sido utilizada popularmente para o auxílio da cicatrização. Amorim e colaboradores (2006) analisaram comparativamente as alterações histológicas proporcionadas pelo uso tópico do extrato aquoso do mesocarpo do babaçu em feridas cutâneas de ratos. Essas feridas foram realizadas com auxilio de punch metálico no dorso dos animais. Seus resultados mostraram que a re-epitelização das feridas foi mais evidente ao $7^{\circ}$ dia de tratamento e houve menor quantidade de células mononucleares aos 14 dias, além de apresentar atividade anti-inflamatória. $\mathrm{O}$ babaçu contribuiu macro e microscopicamente no processo de cicatrização.

A Chenopodium ambrosioides L., conhecida popularmente como "erva de santa maria", pertencente à família Chenopodiaceae ocorre com prevalência em todo território brasileiro (PACIORNIK, 1990 apud RIBEIRO, 2011). Segundo a Organização Mundial da Saúde (OMS), a erva de santa maria é uma das plantas mais utilizadas no mundo inteiro como antiespasmódico, tônico, antirreumático e antipirético, (LORENZI; MATOS, 2002). No Brasil, suas folhas, sementes e sumo são utilizadas popularmente para cura de muitas doenças como: anti-helmíntico, repelente, em contusões, corrimento vaginal, contra gripe, purgante, contra problemas de estômago, úlceras, vermes, e para eliminar pulgas e piolhos (RIBEIRO, 2011). Devido à carência de estudos que comprovassem o seu uso popular no tratamento tópico de feridas cutâneas, Ribeiro (2011) avaliou a eficiência do sumo das folhas e caule da $C$. ambrosioides na contração de feridas cutâneas induzidas no dorso de ratos, com auxílio de tesoura e bisturi. Então, foi concluido que o uso tópico do sumo de C. ambrosioides aumentou o processo de contração das feridas após 12 dias do tratamento.

O Stryphnodendron adstringens Martius, conhecido popularmente como "barbatimão" é uma planta leguminosa do cerrado brasileiro com cascas espessas. Possui um efeito adstringente e a presença de princípios ativos como o tanino, flobafenos e glicídio solúvel. Sua ação farmacológica é dada como cicatrizante de feridas devido à riqueza de taninos (PANIZZA et al., 1988), favorecendo a epitelização e proliferação celular. Outra espécie é a Tabebuia avellanedae, conhecida também como "ipê roxo", oriunda da América do Sul e bastante utilizada na medicina popular (LORENZI, 1992). É utilizado como um poderoso auxiliar no combate a determinados tipos de tumores cancerígenos, tendo reconhecida ação anti-inflamatória, analgésica, antibiótica e antineoplásica. Seus principais princípios ativos são as quinonas, naftoquinonas, taninos e flavonoides (SILVA, 2006). Coelho e colaboradores (2010) estudaram in vivo os extratos bruto da casca do barbatimão e ipê roxo, preparado a partir de extração a quente em água. Foi realizada uma incisão transversal da pele com lâmina de bisturi e posteriormente uma ligadura com fio de poliamida 4-0. O tratamento dos animais foi realizado através de pomada contendo os extratos brutos. O ipê-roxo favoreceu a cicatrização, por sua ação anti-inflamatória, analgésica e antineoplásica, além de favorecer a reepitelização, proliferação vascular e aumento de fibroblastos. Já o extrato de barbatimão devido à sua riqueza de taninos, atuou favorecendo a re-epitelização, proliferação vascular e aumento de fibroblastos. As análises morfológicas realizadas permitiram concluir que o barbatimão e o ipê roxo favorecem o processo cicatricial. A fração acetato de etila do extrato de barbatimão foi estudado também por Hernandes et al. (2010) em feridas cutâneas. Foi observado um aumento significativo no número de metáfases presente no grupo tratado havendo assim um estimulo da proliferação do epitélio.

A Arnica montana L., popularmente conhecida como "arnica", é uma planta herbácea e perene planta pertencente à família Compositae (YUI et al., 1998). Essa espécie é a verdadeira arnica sendo nativa de regiões montanhosas da Europa (LORENZI; MATOS, 2002). É amplamente utilizada na cultura popular como agente anti-inflamatório e cicatrizante, tendo como classe de compostos as lactonas (CUNHA et al., 2003). Nascimento (2011) ao estudar a tintura das flores de arnica incorporada a um gel descobriu que tanto na fase proliferativa quanto na fase de maturação das fibras colágenas, os animais tratados com o gel obtiveram melhor cicatrização quando comparados ao controle, devido a presença de algum princípio ativo em interação com a ferida. Os aspectos celulares foram satisfatórios no processo de cicatrização utilizando $A$. montana. Ainda, os autores atribuem o favorecimento da reparação tecidual a presença das lactonas, helenalina e dihidro-helenalina, que já mostraram possuir atividades anti-inflamatórias (KLAAS et al., 2002).A Aloe vera (L.) Burm. f. conhecida popularmente como "babosa", planta oriunda da África do Sul e da Ásia, pertence à família das Liliaceae, foi introduzida no Brasil no início da colonização, acomodando-se bem em várias regiões do país, principalmente no Nordeste (LORENZI; MATOS, 2002; NEWALL et al., 2002 apud FALEIRO et al., 2009). Vem sendo utilizada há muito tempo como fitoterápico devido as suas ações: antibacteriana, antifúngica, anti-inflamatória, antivirótica e ação cicatrizante (FALEIRO et al., 2009). Possuem em sua composição aloenina, barbaloína e isobarbaloína (KUZUYA et al., 2001). Faleiro e colaboradores (2009) avaliaram o extrato glicólico de Aloe vera na coxa de rato com auxílio de bisturi. As evidências macroscópicas e microscópicas mostraram que o processo de cicatrização foi facilitado pelo fitoterápico, causando contração de feridas. De acordo com Davis (1997), A. vera ao mesmo tempo em que bloqueia a inflamação, também ativa o crescimento dos fibroblastos ocorrendo à cicatrização das feridas.

O Tabernaemontana catharinensis, também conhecida como "jasmim", pode ser encontrada na Argentina, Uruguai, Paraguai, e Sul do Brasil, tendo informação de seu uso como antídoto para mordedura de serpente, calmante e tratamento de verrugas. Na região nordeste da Argentina é muito utilizado para a cura de muitas doenças, como desinfecção de feridas, infecções de garganta, olhos, gonorreia e diarreia causada por parasitas (GUIDA et al., 2001). Janning e colaboradores (2011) avaliaram a cicatrização de feridas cutâneas, com auxílio de bisturi, em ratos tratados com uso tópico do extrato hidroalcoólico de $T$. catharinensis mediante a análise macroscópica e microscópica. Os autores concluíram que o extrato hidroalcoólico possui ação sobre a proliferação vascular, proliferação endotelial e fibroblastos, podendo sugerir então que o extrato de T. catharinensis contém propriedades cicatrizantes.

O Triticum vulgare, também conhecido como "trigo", planta pertencente à família Poaceae. Solórzano et al. (2001) ao testarem um creme vaginal contendo extrato do trigo in vivo mostraram a estimulação do processo de re-epi- 
telização do colo do útero. Souza e colaboradores (2006) estudaram o creme do extrato aquoso de $T$. vulgare (Bandvet ${ }^{\circledR}$ Schering Plough Coopers) em feridas cutâneas, com auxílio de um vazador, experimentalmente induzidas em regiões metacarpiana e lombar dos equinos. As análises macroscópica e microscópica comprovaram que o uso do creme intensificou a migração de células reparadoras, além de estimular a multiplicação dos fibroblastos e produção de colágeno, reduzindo o tempo de cicatrização das feridas.

A Caesalpinia ferrea Martius, conhecida popularmente como "jucá", é uma árvore leguminosa nativa do Brasil, distribuída principalmente no Norte e Nordeste (BRAGANÇA, 1996). Vem sendo utilizada popularmente no Rio Grande do Norte como cicatrizante de feridas. Oliveira e colaboradores (2010) estudaram as cascas de C. ferrea na cicatrização de feridas cutâneas abertas de caprinos. As feridas foram realizadas lado a lado na região torácica dos caprinos com remoção da pele. Foi evidenciada atividade anti-inflamatória e antimicrobiana obtendo um efeito significativo na cicatrização da pele de caprinos. Foi observada ausência de secreção na ferida, possivelmente devida à formação de película pela presença de taninos.

A Calendula officinalis, conhecida popularmente como "calêndula", pertence à família Asteraceae e tem sido usada medicinalmente desde o século 12 (MOLINA et al., 2008). No Brasil, é muito utilizada com fins ornamentais no sul do país (LORENZI; MATOS, 2002). As flores e folhas da calêndula foram utilizadas na guerra civil americana para tratar os feridos devido ao seu poder cicatrizante, anti-inflamatório e antisséptico. A principal atividade da calêndula foi a ação anti-inflamatória, atribuída aos triterpenoides e glicosídeos (DELLA LOGGIA et al., 1994). Os terpenos oxigenados podem ser os responsáveis pela atividade antimicrobiana (GRACZA, 1987).

O Helianthus annus, conhecido popularmente por "girassol", originou-se nos Estados Unidos e México, até o século XVIII cultivava-se como planta medicinal e ornamental, só no século XX começou a ser usado como fonte de óleo comestível na Rússia. São Paulo e Paraná são os estados brasileiros que tem o maior potencial para o cultivo, pois é necessário muito sol e umidade (TESKE; TRENTINI, 1997 apud WENDT, 2005). Nas flores de girassol, diversos corantes, glicosídeos e flavonoides, foram encontrados e, no óleo das sementes são encontrados o ácido oleico e os ácidos graxos não saturados, especialmente o ácido linoleico. O óleo de girassol favorece o aparecimento de um tecido de granulação mais fino, ou seja, propicia uma recuperação epidérmica. $\mathrm{O}$ ácido linoleico tem propriedades pró-inflamatórias que estimulam a neovascularização (angiogênese) local e consequentemente a migração celular, proliferação e diferenciação fibroblástica e também a síntese de matriz extracelular (MARQUES et al., 2004). Wendt (2005) estudou comparativamente a eficiência da calêndula e do óleo de girassol em feridas cutâneas de coelhos, realizadas com auxílio de um bisturi. Concluíram que o extrato das flores da calêndula e o óleo extraído dos grãos aplicados junto possui ação anti-inflamatória e antibacteriana, mostrando assim a sua eficácia na cicatrização de feridas com processo infeccioso presente.

O Schinus terebinthifolius Raddi, conhecido popularmente por "aroeira", é uma planta pertencente à família Anacardiaceae, comum da vegetação litorânea dos Estados nordestinos brasileiros. Sua casca é indicada como anti-inflamatório e cicatrizante (CARMELLO-GUERREIRO; PAOLI, 1999). Nunes e colaboradores (2006) avaliaram o extrato de hidroalcóolico da entrecasca de $S$. terebinthifolius na linea alba de ratos. Foi realizada uma incisão longitudinal mediana iniciando-se logo abaixo do processo xifoide estendendo-se inferiormente por 3,0 cm compreendendo a pele e o subcutâneo, identificando-se a linea alba. Foi descoberto que o extrato apresentou ação anti-inflamatória e foi evidenciada a ação cicatrizante, uma vez que houve aumento dos fibroblastos na região da sutura.

O Catharanthus roseus L, popularmente conhecido como "vinca rósea", é nativa da Bacia do Caribe e tem sido utilizado popularmente para tratar uma ampla variedade de doenças, incluindo diabetes. Nayak e Pereira (2006) estudaram as flores da C. roseus, em feridas cutâneas de ratos, realizadas com auxílio de bisturi. Foi utilizado o extrato etanólico que apresentou aumento na contração de ferida, aumento na concentração de hidroxiprolina e atividade antibacteriana contra Pseudomonas aeruginosa e Staphylococcus aureus.

Coronopus didymus, conhecido popularmente com "mastruz", é muito utilizado como agente cicatrizante em feridas cutâneas. Nitz e colaboradores (2006) estudaram comparativamente, através de metodologia histométrica, o potencial cicatrizante do extrato aquoso de $C$. didymus. Foi realizado uma epilação em ratos, de toda a área na região dorso costal, em seguida foi demarcado com auxílio de uma caneta hidrocor, uma área circular de $1 \mathrm{~cm}$ de diâmetro, utilizou-se uma tesoura para seccionar um fragmento cutâneo, correspondendo a área demarcada, até a exposição da fáscia muscular, os animais foram tratados com o extrato aquoso de C. didymus e descobriram a eficiência no processo cicatricial de feridas cutâneas em ratos, promovendo um aumento no número de fibroblastos e fibras colágenas.

O Quadro 1, abaixo, traz uma representação das plantas estudadas, características analisadas e classe das substâncias presentes. 
Quadro 1: Representação das plantas estudadas e características analisadas.

\begin{tabular}{|c|c|c|c|c|c|}
\hline Nome Científico & $\begin{array}{l}\text { Nome } \\
\text { Popular }\end{array}$ & $\begin{array}{c}\text { Parte da } \\
\text { planta usada }\end{array}$ & $\begin{array}{l}\text { Extração/ } \\
\text { utilização }\end{array}$ & $\begin{array}{c}\text { Classe das } \\
\text { substâncias }\end{array}$ & Efeito da cicatrização \\
\hline $\begin{array}{l}\text { Aloe vera }(\mathrm{L} .), \\
\text { Burm. f. }\end{array}$ & Babosa & Folhas & Extrato glicólico & Polissacarídeo & $\begin{array}{c}\text { Contração de feridas } \\
\text { (FALEIRO et al., 2009) }\end{array}$ \\
\hline $\begin{array}{c}\text { Arnica montana } \\
\text { L. } \\
\end{array}$ & Arnica & Flores & Gel & $\begin{array}{c}\text { Éteres helenalina } \\
\text { Dihidro-helenalina }\end{array}$ & $\begin{array}{l}\text { Favorece a cicatrização } \\
\text { (NASCIMENTO, 2011) }\end{array}$ \\
\hline $\begin{array}{c}\text { Caesalpinia férrea } \\
\text { Martius }\end{array}$ & Jucá & Cascas & Pomada & Taninos & $\begin{array}{c}\text { Favorece a cicatrização, Atividade } \\
\text { anti-inflamatória } \\
\text { (OLIVEIRA et al., 2010) }\end{array}$ \\
\hline $\begin{array}{l}\text { Calendula } \\
\text { officinalis }\end{array}$ & Calêndula & Flores & Pomada & $\begin{array}{l}\text { Triterpenoides } \\
\text { Glicosídeos } \\
\text { Terpenos } \\
\text { oxigenados }\end{array}$ & $\begin{array}{c}\text { Eficaz na cicatrização de feridas com } \\
\text { processo infecioso } \\
\text { (WENDT, 2005) }\end{array}$ \\
\hline $\begin{array}{l}\text { Catharanthus } \\
\text { roseus } \mathrm{L} .\end{array}$ & Vinca Rosea & Flores & Extrato etanólico & $\begin{array}{c}\text { Triterpenóides } \\
\text { Taninos Alcalóides }\end{array}$ & $\begin{array}{l}\text { Aumento do teor de hidroxiprolina, } \\
\text { contração e tensão da ferida, } \\
\text { Atividade antimicrobiana } \\
\text { (NAYAK; PEREIRA, 2006) }\end{array}$ \\
\hline $\begin{array}{l}\text { Chenopodium } \\
\text { ambrosioides L. }\end{array}$ & $\begin{array}{c}\text { Erva de Santa } \\
\text { Maria }\end{array}$ & Folhas e caule & $\begin{array}{c}\text { Sumo com água } \\
\text { destilada }\end{array}$ & - & $\begin{array}{c}\text { Aumento da contração das feridas, } \\
\text { Ação antimicrobiana Anti- } \\
\text { inflamatória } \\
\text { (RIBEIRO, 2011) }\end{array}$ \\
\hline $\begin{array}{l}\text { Coronopus } \\
\text { didymus }\end{array}$ & Mastruz & - & Extrato aquoso & $\begin{array}{c}\text { Flavonoides } \\
\text { Taninos Saponinas }\end{array}$ & $\begin{array}{c}\text { Aumento do número de fibroblasto e } \\
\text { fibras colágenas } \\
\text { (NITZ et al., 2006) }\end{array}$ \\
\hline Helianthus annus & Girassol & Grãos & Pomada & Ácido linoleico & $\begin{array}{l}\text { Propriedades pró-inflamatórias, } \\
\text { Estímulo da neovascularização, } \\
\text { migração celular, proliferação e } \\
\text { diferenciação fibroblástica e também } \\
\text { a síntese de matriz extracelular } \\
\text { (WENDT, 2005) }\end{array}$ \\
\hline $\begin{array}{l}\text { Orbignya } \\
\text { phalerata }\end{array}$ & Babaçu & Mesocarpo & Extrato aquoso & $\begin{array}{l}\text { Carboidratos Sais } \\
\text { minerais }\end{array}$ & $\begin{array}{l}\text { Contribuição positiva para o } \\
\text { processo da cicatrização, Ação anti- } \\
\text { inflamatória } \\
\text { (AMORIM et al., 2006) }\end{array}$ \\
\hline $\begin{array}{l}\text { Schinus } \\
\text { terebinthifolius } \\
\text { Raddi }\end{array}$ & Aroeira & Entrecasca & $\begin{array}{c}\text { Extrato } \\
\text { hidroalcoólico }\end{array}$ & - & $\begin{array}{c}\text { Aumento de fibroblastos, Resistência } \\
\text { de suturas } \\
\text { (NUNES et al., 2006) }\end{array}$ \\
\hline $\begin{array}{l}\text { Stryphnodendron } \\
\text { adstringens }\end{array}$ & Barbatimão & Casca & $\begin{array}{l}\text { Solução aquosa } \\
\text { extraída a quente/ } \\
\text { pomada }\end{array}$ & $\begin{array}{l}\text { Tanino Flobafenos } \\
\text { Glicídio solúvel }\end{array}$ & $\begin{array}{c}\text { Favorecem a cicatrização } \\
\text { (COELHO et al., 2010) }\end{array}$ \\
\hline $\begin{array}{l}\text { Tabebuia } \\
\text { avellanedae }\end{array}$ & Ipê roxo & Casca & $\begin{array}{l}\text { Solução aquosa } \\
\text { extraída a quente/ } \\
\text { pomada }\end{array}$ & $\begin{array}{l}\text { Quinonas } \\
\text { Naftoquinona } \\
\text { Taninos } \\
\text { Flavonoides }\end{array}$ & $\begin{array}{c}\text { Favorecem a cicatrização } \\
\text { (COELHO et al., 2010) }\end{array}$ \\
\hline $\begin{array}{l}\text { Tabernaemontana } \\
\text { catharinensis }\end{array}$ & Jasmim & Folhas & $\begin{array}{c}\text { Extrato } \\
\text { hidroalcoólico }\end{array}$ & $\begin{array}{l}\text { Flavonoides } \\
\text { Taninos }\end{array}$ & $\begin{array}{l}\text { Maior contração na ferida } \\
\text { (JANNING et al. 2011) }\end{array}$ \\
\hline Triticum vulgare & Trigo & Espiga & $\begin{array}{l}\text { Extrato aquoso/ } \\
\text { creme }\end{array}$ & Fitoestimulinas & $\begin{array}{c}\text { Migração de células reparadoras, } \\
\text { Estimulação de fibroblastos, Produção } \\
\text { de colágeno } \\
\text { (SOUZA et al., 2006) }\end{array}$ \\
\hline
\end{tabular}

\section{Considerações Finais}

Muitas plantas são utilizadas empiricamente pela população com a finalidade cicatrizante. Por meio de uma busca na literatura científica foram encontradas diversas plantas da flora brasileira, ditas popularmente eficazes na cicatrização de feridas, cuja comprovação foi obtida através de testes in vivo.

Nesses trabalhos analisados, pode-se observar que todas as plantas elencadas favoreceram o processo de cicatrização por meio de diferentes efeitos, tais como anti-inflamatório, antimicrobiano, estimulação da proliferação, migração e atividade celular. Essa atividade pode estar relacionada com as diferentes classes de substâncias encontradas em cada planta. Entretanto, é importante a determinação do mecanismo de ação dessas plantas para complementar os resultados obtidos. 


\section{Referências}

AMORIM, E. et al. Efeito do uso tópico do extrato aquoso de Orbignya phalerata (babaçu) na cicatrização de feridas cutâneas: estudo controlado em ratos. Acta Cir. Bras. v. 21, suppl. 2, p. 67-76, 2006.

\section{AMOROZO, M. C. M.; GELY, A. Uso de plantas medicinais por caboclos do Baixo Amazonas. Barcarena: Museu Paraense Emílio Goeldi, 1988. 47 p.}

BALBINO, C. A.; PEREIRA, L. M.; CURI, R. Mecanismos envolvidos na cicatrização: uma revisão. Rev. Bras. de Cienc. Farm. v. 41, n. 1, p. 27-51, 2005.

BRAGANÇA, L. A. R. Plantas medicinais antidiabéticas: uma abordagem multidisciplinar. Niterói: EDUFF, 1996. $300 \mathrm{p}$.

CANDIDO, L. C. Novas abordagens no tratamento de feridas. São Paulo: Senac, 2001. 282 p.

CARMELLO-GUERREIRO, S. M.; PAOLI,

A. A. S. Morfologia e anatomia da semente de Schinus terebinthifolius Raddi (Anacardiaceae) em desenvolvimento. Rev. Bras. Bot. v. 22, n. 1, p.1-12, 1999

CARNEIRO, S. C. S.; RAMOS-E-SILVA, M. Cicatrização. In: KEDE, M. P. V.; SABATOVICHE, O. Dermatologia estética. São Paulo: Atheneu, 2007. 11-14 p.

COELHO, J. M. et al. O efeito da sulfadiazina de prata, extrato de ipê-roxo e extrato de barbatimão na cicatrização de feridas cutâneas em ratos. Rev. Col. Bras. Cir. v. 37, n. 1, p. 45-51, 2010.

CUNHA, A. P. da; SILVA, A. P. da; ROQUE, O. R. Plantas e produtos vegetais em fisioterapia. Lisboa: Fundação Calouste Gulbenkian, 2003.

CUTTLE, L. et al. Collagen in the scarless fetal skin wound: Detection with Picrosirius-polarization. Wound Repair Regen, v. 13, n. 2, p. 198-204, 2005.

DAVIS, R. H. Aloe vera: a scientific approach. New York: Vantage Press. 1997. In: FALEIRO, C. C. et al. O extrato das folhas de babosa, Aloe vera na cicatrização de feridas experimentais em pele de ratos, num ensaio controlado por placebo. Natureza on line, v. 7, p. 56-60, 2009.

DELLA LOGGIA, R. et al. The role of triterpenoids in the tropical anti-inflammatory activity of Calendula officinalis flower. Planta Med. v. 60, n. 6, p. 516-520, 1994.

DIEGELMANN, R. F.; COHEN, I. K.; KAPLAN, A. M. The role of macrophages in wound repair: a review. Plast Reconstr Sur. v. 68, n. 1, p. 107-113, 1981.

FALEIRO, C. C. et al. O extrato das folhas de babosa, Aloe vera na cicatrização de feridas experimentais em pele de ratos, num ensaio controlado por placebo. Natureza on line, v. 7, n. 2, p. 56-60, 2009

KLAAS, C. A. et al. Studies on the anti-inflammatory activity of phytopharmaceuticals prepared from arnica flowers. Planta Med. v. 68, n. 5, p. 385-391, 2002.

GRACZA, L. Oxygen-containing terpene derivatives from Calendula officinalis. Planta Med. v. 53, n. 2, p. 227, 1987.

GRINNELL, F.; BILLINGHAN, R.; BURGESS, L. Distribution of fibronectin during wound healing in vivo. J Investig Dermatol. v. 76, n. 3, p. 181-189, 1981.

GUIDA, A. et al. Staphylococcus aureus meticilinoresistentes, sensible a extractos de Tabernaemontana catharinensis A.DC. Acta Farm. Bonaer. v. 20, n. 3, p. 205-208, 2001.

HERNANDES, L. et al. Wound-healing evaluation of ointment from Stryphnodendron adstringens (barbatimão) in rat skin. Braz. J. Pharm. Sci. v. 46, n. 3, p. 431-436, 2010 .

JANNING, D.; ALBUQUERQUE, C. A. C.; BARAUNA, S. C. Avaliação preliminar do extrato hidroalcoólico de Tabernaemontana catharinensis no processo de cicatrização de feridas em pele de ratos (Rattus norvegicus). Rev.

Eletrônica Farm. v. 8, n. 3, p. 53-64, 2011.

KUZUYA, H. et al. Determination of aloenin, barbaloin and isobarbaloin in Aloe species by micellar electrokinetic chromatography. J Chromatogr B Biomed Sci Appl. v. 752, n. 1, p. 91-97, 2001.

LORENZI, H. Árvores brasileiras: manual de identificação e cultivo de plantas arbóreas do Brasil. Nova Odessa: Plantarum, 1992.

LORENZI, H.; MATOS, F. J. de A. Plantas medicinais no Brasil: nativas e exóticas. 2. ed. Nova Odessa: Instituto Plantarum, 2008. 544 p.

LUSSIGNOLI, S. et al. Effect of Traumeel S, a homeopathic formulation, on blood-induced inflammation in rats. Complement Ther Med. v. 7, n. 4, p. 225-230, 1999.

MANDELBAUM, S. H.; DI SANTIS, É. P.; MANDELBAUM, M. H. S. Cicatrização: conceitos atuais e recursos auxiliares - Parte I. An Bras Dermatol. v. 78, n. 4, p. 393-410, 2003.

MARQUES, S. R. et al. The effects of topical application of sunflower-seed oil on open wound healing in lambs. Acta Cir. Bras. v. 19, n. 3, p. 196-209, 2004.

MOLINA, F. P. et al. Própolis, sálvia, calêncula e mamonaatividade antifúngica de extratos naturais sobre cepas de Candida albicans. Braz. Dent. Sci. v. 11, n. 2, p. 86-93, 2008. 
NASCIMENTO, E. D. M. Estudo do efeito da Arnica (Arnica montana L.) feridas cutâneas em ratos sobre a cicatrização. 2011. 24. Monografia Faculdade Ciências Médicas e da Saúde, Pontifícia Universidade Católica, São Paulo, 2011.

NAYAK, B. S.; PEREIRA, L. M. P. Catharanthus roseus flower extract has wound-healing activity in Sprague Dawley rats. BMC Complement Altern Med. v. 6, n. 41, p. 1-6, 2006.

NEWALL, C. A.; ANDERSON, L. A.; FHILLIPSON, J. D. Plantas medicinais: guia para profissionais da saúde. São Paulo: Premier, 2002.

NITZ, A. C. et al. Estudo morfométrico no processo de cicatrização de feridas cutâneas em ratos, usando: Coronopu didymus e Calendula officinalis. ACM Arq. Catarin. Med. v. 35, n. 4, p. 74-79, 2006.

NUNES JUNIOR, J. A.T. et al. Avaliação do efeito do extrato hidroalcoólico de Schinus terebinthifolius Raddi (Aroeira) no processo de cicatrização da linea alba de ratos. Acta Cir. Bras. v. 21, suppl. 3, p. 8-15, 2006.

OLIVEIRA, A. F. et al. Avaliação da atividade cicatrizante do jucá (Caesalpinia ferrea Mart. ex Tul. var. ferrea) em lesões cutâneas de caprinos. Rev. bras. plantas med. v. 12, n. 3, p. 302-310, 2010.

OVALLE, W. K.; NAHIRNEY, P. C. Netter bases da histologia. Rio de Janeiro: Elsevier, 2008.

PACIORNIK, E. F. A planta nossa de cada dia: plantas medicinais: descrição e uso. Curitiba: Gráfica Copygraf, 1990. 92 p.

PANIZZA, S. et al. Stryphnodendron barbadetiman (Vellozo) Martius: teor em tanino na casca e sua propriedade cicatrizante. Rev. Ciênc. Farm. v. 10, p.101$106,1988$.

RIBEIRO, R. V. Influência do sumo de Chenopodium ambrosioides L. (erva de Santa Maria) na contrações de feridas cutâneas induzidas em dorso de ratos da linhagem Wistar. Connectionline, n. 3, 2008.

ROTTA, O. Guia de dermatologia: clínica, cirúrgica e cosmética. São Paulo: Manole, 2008. p. 3-6.

SILVA, B. P.; PARENTE, J. P. An anti-inflamatory and immunomo-dulatory polysaccharide from Orbignya phalerata. Fitoterapia, v. 72, n. 8, p. 887-893, 2001.

SILVA, C. M. P.; ROCH, R. M.; MORENO, J. S. O babaçu (Orbignya phalerata) como provável fator de risco de infecção humana pelo agente de cromoblastomicose no estado do Maranhão. Rev. Soc. Bras. Med. Trop. v. 28, n. 1, p. 49-52, 1995.

SILVA, D. T. O uso do Ipê-Roxo (Tabebuia vellanedae) na cicatrização de feridas cutâneas em ratos. 2006. Dissertação (Mestrado em Ciência Animal nos Trópicos) - Universidade Federal da Bahia, Salvador, 2006.

SINGER, A. J.; CLARK, R. A. F. Cutaneous wound healing. N Engl J Med., v. 341, n. 10, p. 738-746, 1999.

SOLÓRZANO, O. T. et al. Evaluación de la actividad reepitelizante del Triticum vulgare en la cervicitis crónica erosiva. Rev Fac Med UNAM, v. 44, n. 1, 2001.

SOUZA, D. W. et al. Ensaio da aplicação de creme à base de Triticum vulgare na cicatrização de feridas cutâneas induzidas em equinos. Rev. Bras. Pl. Med. v. 8, n. 3, p. 9-13, 2006.

TESKE, M.; TRENTINI, A. M. M. Compêndio de fitoterapia, 4. ed. Curitiba: Herbarium; Laboratório Botânico, 1997.

WENDT, S. B. T. Comparação da eficácia da calêndula e do óleo de girassol na cicatrização por segunda intenção de feridas em pequenos animais. 2005. 85 f. Dissertação (Mestrado em Ciências Veterinárias) - Universidade Federal do Paraná, Curitiba, 2005.

YUI, F.; LINARELLI, M. C. B.; ZELANTE, P. M. Atividade antiinflamatória de Arnica montana. Rev. Ciênc. Méd. v. 7, n. 1, p. 21-26, 1998. 